\title{
A randomized controlled trial comparing lifestyle intervention to letrozole for ovulation in women with polycystic ovary syndrome: a study protocol
}

\author{
Dylan A. Cutler ${ }^{1}$, Alana K. Shaw ${ }^{2}$, Sheila M. Pride ${ }^{1}$, Mohamed A. Bedaiwy ${ }^{1}$ and Anthony P. Cheung ${ }^{1,2^{*}}$ (D)
}

\begin{abstract}
Background: Polycystic ovary syndrome (PCOS) affects between 8 and 18\% of women and is the leading cause of female anovulatory infertility. Unfortunately, common treatments for women trying to conceive can be ineffective as well as disruptive or harmful to patients' quality of life. Despite evidence that women with PCOS have expressed the need for alternative fertility treatments, lifestyle interventions incorporating a nutritional plan with supplementation, increased physical activity, and techniques for stress management have not been combined as a program and studied in this population. Literature suggests that each of these individual components can positively influence reproductive hormones and metabolic health.

Methods/design: This is a randomized controlled trial which will include 240 women diagnosed with PCOS, according to the Rotterdam criteria, who are trying to conceive. Participants will be randomized to either a comprehensive lifestyle intervention program or prescribed an oral fertility medication, letrozole. These two groups will be further randomized to consume either myo-inositol or a placebo. Participants will be between the ages of 18 and 37 years. Exclusion criteria include women who have already begun fertility treatment, who are currently using myo-inositol or have taken it within the past 3 months, or who are being treated for, or have a history of, an eating disorder. The primary outcome will be the ovulation rate, the secondary outcome will be conception. Other outcomes include miscarriage rates, validated rating measures of overall quality of life (including social, relational, mind/body and emotional sub-categories) and mental health scores (depression, anxiety, and stress).
\end{abstract}

Discussion: This trial will determine the effectiveness of a structured lifestyle-based comprehensive intervention program for women with PCOS experiencing infertility. In addition, it will determine whether supplementing with myo-inositol provides any further benefit. The objective of this study is to assess a possible non-pharmacological solution to ovulatory dysfunction in these patients and perhaps improve other associated features of PCOS.

Trial registration: ClinicalTrials.gov, ID: NCT02630485. Registered on 15 December 2015.

Keywords: Polycystic ovary syndrome, Ovulation, Lifestyle, Infertility

\footnotetext{
* Correspondence: apcheung@fertilitywithgrace.com

${ }^{1}$ Division of Reproductive Endocrinology and Infertility, Department of

Obstetrics and Gynaecology, University of British Columbia, Vancouver, BC,

Canada

${ }^{2}$ Grace Fertility and Reproductive Medicine, 210-604 West Broadway,

Vancouver, BC V5Z 1G1, Canada
}

(c) The Author(s). 2018 Open Access This article is distributed under the terms of the Creative Commons Attribution 4.0 International License (http://creativecommons.org/licenses/by/4.0/), which permits unrestricted use, distribution, and reproduction in any medium, provided you give appropriate credit to the original author(s) and the source, provide a link to the Creative Commons license, and indicate if changes were made. The Creative Commons Public Domain Dedication waiver (http://creativecommons.org/publicdomain/zero/1.0/) applies to the data made available in this article, unless otherwise stated. 


\section{Background}

Polycystic ovary syndrome (PCOS) is a heterogenous endocrine syndrome characterized by chronic anovulation and hyperandrogenism (clinical or biochemical) according to the original $1990 \mathrm{NIH}$ criteria. The 2003 Rotterdam criteria added the presence of polycystic ovarian morphology on ultrasound which resulted in four distinct PCOS phenotypes. PCOS is also associated with obesity, insulin resistance, metabolic syndrome, reduced quality of life, increased anxiety and higher rates of depression [1]. Between 70 and $80 \%$ of women with PCOS have difficulty conceiving due to oligo- or anovulation. Despite ovulation rates of $75-80 \%$ achieved with the use of ovulation-induction agents and a conception rate per cycle of $22 \%$ (comparable to that of a healthy women trying to conceive naturally in their first year), some women with PCOS remain unresponsive $[2,3]$. In contrast, evidence suggests that lifestyle (diet, activity and stress management) influences ovulation and the effectiveness of fertility treatments [4-6].

While lifestyle intervention is recommended as first-line management for women with PCOS and obesity, $45 \%$ of women with PCOS reported that they have never received information on lifestyle management and only $61-76 \%$ of reproductive endocrinology and infertility obstetric gynecologists (REI-ObGyn) and 46-61\% of obstetric gynecologists (ObGyn) recommended lifestyle modifications to patients for fertility or non-fertility problems $[7,8]$. Even with efforts to improve dietary and exercise habits, women with PCOS often have difficulty managing their weight. In addition, $62 \%$ of women with PCOS reported never receiving information about emotional support or counseling [7]. It appears that attention to general physical and psychological health is inconsistent at the clinical level yet recognized as an important factor in the effective management of women receiving the life-altering diagnosis of PCOS and/or infertility $[9,10]$.

A survey of 657 women with PCOS found that $99 \%$ of participants would prefer to use alternative methods of treatment than those usually prescribed by their physicians such as clomiphene or oral contraceptives [11]. More recently a literature review by Arentz et al., reported that $70 \%$ of women with PCOS use a variety of complementary and alternative medicines, such as vitamin, mineral and herbal supplements. While the most common reason given was to "treat PCOS," other reasons included "to treat infertility," "to improve general well-being," and "to reduce depression" [12].

There have been no studies to date evaluating the reproductive outcomes of a comprehensive, three-component, intervention program incorporating structured nutritional and physical activity combined with techniques for stress management in women with PCOS trying to conceive [13]. The literature shows that women with PCOS can significantly benefit from lifestyle changes, specifically, eating a low-glycemic diet, incorporating nutritional supplements, increasing their activity level, and managing stress [14-17]. However, prior lifestyle intervention studies have limitations including high drop-out rates, lack of defining specific PCOS phenotypes, and small cohort sizes. In addition, most studies have focused on only one lifestyle-related change, such as diet, as opposed to a more synergistic approach wherein diet, exercise, and stress reduction are combined. In addition, the implementation of a mind/body program to help reduce stress which could potentially increase ovulation and conception has yet to be studied in a PCOS cohort. Cognitive behavioural therapy has been implemented in infertile populations for over 30 years [18]. These programs teach a variety of coping skills such as learning relaxation techniques, stress management, and provide group support. The relaxation response is a powerful tool already proven effective in stress-related diseases such as cancer, cardiovascular disease, and mental disorders. Since women with PCOS have a higher prevalence of mental health conditions, inducing the relaxation response through mindfulness training could prove an effective stress management strategy for our study cohort [19].

In addition to a low-glycemic diet, physical activity, and stress reduction, a diet supplemented with myo-inositol may provide further hormonal homeostasis and improve metabolic functioning [20]. Myo-inositol is one of nine stereoisomers of inositol and sometimes considered a member of the B complex vitamin family, albeit humans can synthesize this isomer from glucose. It acts as a second messenger in insulin signaling pathways. Several studies have shown that insulin resistance with its compensatory hyperinsulinemia play a pivotal role in both the metabolic and ovarian hormone dysfunction observed in PCOS women. There is evidence that women with PCOS and insulin resistance could be deficient in myo-inositol which would impact glucose metabolism. In addition, myo-inositol levels in follicular fluid are lower in PCOS women with hyperinsulinemia compared to healthy women [21, 22]. An earlier study described that higher levels of myo-inositol in follicular fluid correlated with oocyte quality and maturity [23]. When supplemented with myo-inositol, women with PCOS have experienced reduced serum insulin, testosterone, and increased rates of ovulation [24, 25]. Myo-inositol has also been shown to improve oocyte number and quality [26].

We hypothesize that implementing a comprehensive program of lifestyle changes in women with PCOS will help restore ovulation by weight loss, reduce serum androgen levels and increase sensitivity to insulin. A lifestyle change program could also ameliorate hirsutism over time. Such changes should improve overall 
psychological well-being and quality of life. Finally, we expect that distinct phenotypes of PCOS will respond differently to lifestyle intervention. Such information will allow us to better determine which phenotypes are best served using our comprehensive approach, and to identify more specifically those factors affecting outcomes.

The objectives of this study are: to determine whether the comprehensive lifestyle intervention described is effective in restoring ovulation and compare this to letrozole; to determine whether the addition of myo-inositol improves ovulation rates, insulin resistance, metabolic parameters; and finally to evaluate differences in the responses for each of four main PCOS phenotype sub-groups.

\section{Methods/design}

\section{Overall study design}

Women diagnosed with PCOS will be recruited at the Grace Fertility and Reproductive Medicine Centre in Vancouver, Canada. This study follows a factorial design (Fig. 1). Participants will be randomized with a 1:1 allocation into two groups: the first group participating in a comprehensive lifestyle intervention program provided by our clinic ("Graceful Lifestyle Changes" (GLC) program) while the second group will be prescribed letrozole to induce ovulation, with the standard physician counseling (Fig. 2). Within each group, participants will be further randomized with a 1:1 allocation to either receive oral myo-inositol or a placebo. Block randomization will be performed at both stages using a computer-generated random numbers table and the software program REDCap. All clinicians, research scientists and participants involved in the study will be blinded to block sizes and allocation of myo-inositol/placebo. A researcher not involved in the study will be responsible for the randomization procedures. A data monitoring committee will not be necessary given the known safety of the proposed interventions. The SPIRIT 2013 Checklist for trial protocols has been included (see Additional file 1).

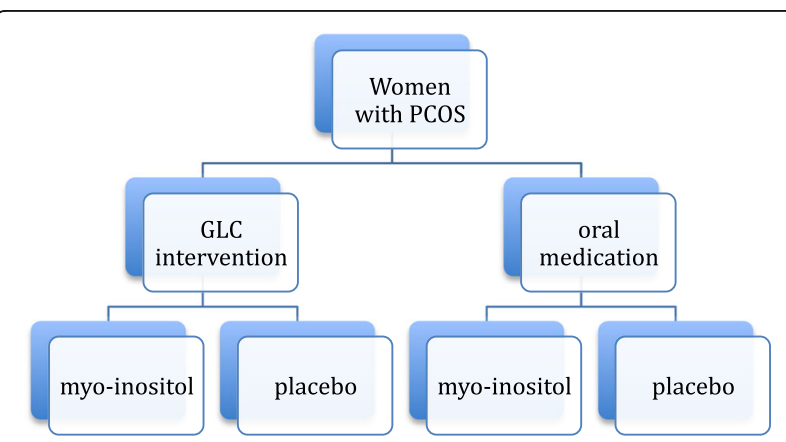

Fig. 1 Participants will be randomized into two groups: the "Graceful Lifestyle Changes" (GLC) group and the oral medication group. Both groups will be further randomized to receive either myo-inositol or its placebo

\section{Lifestyle intervention group (GLC)}

The GLC group will have weekly check-ins with physicians and educators for 12 consecutive weeks. A wellness booklet designed and provided by clinicians will outline the main concepts being taught. Each week, subjects will receive detailed comprehensive instructions on how to follow a low-glycemic meal plan, incorporate walking 10,000 steps a day, and induce the relaxation response using cognitive behavioural therapy techniques. The low-glycemic-meal plan requires participants to consume $45 \%$ of carbohydrates in their diet and no more than $55 \mathrm{~g}$ of glycemic load a day, which is similar to previous low-glycemic diet intervention studies. Lists will be provided outlining foods that are low, medium, or high in their glycemic load. Hence, within their overall meal plan, participants will be advised to eat predominantly low-glycemic foods and to limit high-glycemic foods. They will also be provided with a pedometer to record the number of steps taken each day. These steps can be achieved by any form of exercise that participants can sustain. Lastly, participants will practice relaxation response exercises for $20 \mathrm{~min}$ daily on their own, in combination with 6 weeks of mindfulness training provided within the 12-week intervention program.

\section{Data collection}

Participants will complete a 3-day food diary report during the baseline, 4th, 8th, and 12th weeks. Participants will also receive a phone call from a trained researcher to complete a 24-h diet recall at the end of the 2nd, 6th, and 10th weeks. This recall will assess all food consumed in the previous $24 \mathrm{~h}$. These combined methods will aim to assess overall compliance to the low-glycemic diet, provide researchers with information on the length of time that participants take to comply, evaluate any fluctuations in eating habits and compare these two types of nutritional assessments.

Participants will also record their physical activity during the baseline, 4th, 8th, and 12th weeks based on their pedometer recordings and will record the meditation and relaxation exercises performed daily.

Compliance to the physical activity portion will be measured through daily pedometer readings recorded by the participant. Compliance will be ensured additionally through weekly check-ins/weigh-ins and frequent motivational email reminders.

Psychological well-being (depression, anxiety, stress, and quality of life) will be assessed before and after the 12-week intervention by two validated self-report questionnaires: the Depression Anxiety Stress Scale (DASS) and the Fertility-Related Quality of Life tool (FertiQoL) [27, 28].

\section{Letrozole group}

The use of clomiphene citrate has been commonly used to treat anovulatory infertility. However, clinical experience 


\begin{tabular}{|c|c|c|c|c|c|c|}
\hline & Enrolment & Allocation & \multicolumn{4}{|c|}{ Post-allocation } \\
\hline TIMEPOINT & $\begin{array}{c}\text { Recruitment } \\
\text { Stage }\end{array}$ & $\begin{array}{c}\text { Pre- } \\
\text { intervention }\end{array}$ & Cycle 1 & Cycle 2 & Cycle 3 & $\begin{array}{c}\text { Post- } \\
\text { intervention }\end{array}$ \\
\hline \multicolumn{7}{|l|}{ ENROLMENT: } \\
\hline Eligibility screen & $x$ & & & & & \\
\hline Informed consent & $\mathrm{X}$ & & & & & \\
\hline Allocation & & $\mathrm{X}$ & & & & \\
\hline \multicolumn{7}{|l|}{ INTERVENTIONS: } \\
\hline \multicolumn{7}{|l|}{ Letrozole } \\
\hline \multicolumn{7}{|l|}{ GLC } \\
\hline \multicolumn{7}{|l|}{ Myo-inositol } \\
\hline \multicolumn{7}{|l|}{ Placebo } \\
\hline \multicolumn{7}{|l|}{ ASSESSMENTS: } \\
\hline \multirow{2}{*}{$\begin{array}{r}\text { Anthropometrics } \\
\text { Dietary Intake }\end{array}$} & X & $\mathrm{X}$ & $\mathrm{X}$ & X & X & X \\
\hline & & $x$ & $x$ & 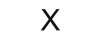 & $x$ & \\
\hline \multirow{2}{*}{ Physical Activity } & & $\mathrm{X}$ & 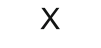 & X & $x$ & \\
\hline & & $x$ & $x$ & $x$ & $x$ & \\
\hline Meditation Exercises & & & $\mathrm{X}$ & $\mathrm{X}$ & $x$ & $\mathrm{X}$ \\
\hline Ovulation & & & $\mathrm{X}$ & $\mathrm{X}$ & $x$ & $x$ \\
\hline Conception & & $x$ & & & & $x$ \\
\hline Depression & & $x$ & & & & $x$ \\
\hline Anxiety & & $x$ & & & & $x$ \\
\hline Quality of Life & & $x$ & & & & $\mathrm{X}$ \\
\hline
\end{tabular}

Fig. 2 Schedule of enrolment, interventions, and assessments

and recent study evidence has suggested that letrozole is more effective than clomiphene citrate in achieving ovulation in PCOS [29]. Additionally, letrozole does not negatively affect endometrial thickness. The initial dose will be $5 \mathrm{mg}$ daily for 5 days which can be increased to a total daily dose of $7.5 \mathrm{mg}$, if necessary, depending on the ovulatory response as per current clinical practice at the Grace Fertility Centre. The first dose will be defined as "cycle day 3 " which corresponds to cycle day 3 of spontaneous menses or day 3 of an induced withdrawal bleed following progestogen withdrawal. This treatment regimen will continue for three cycles or until pregnancy is achieved. Subjects will be advised to have intercourse every other day during the expected ovulatory period (beginning cycle day 12, for a week) and to use urinary ovulation prediction kits.
Myo-inositol group

Participants will be instructed to ingest $6 \mathrm{~g}$ of myo-inositol (or its placebo) in water each morning for 12 weeks.

\section{Outcomes}

Ovulation is the primary outcome (categorical "yes" or "no") and frequency of ovulation (nominal "0", " 1 ", " 2 ", or "3") during the 12-week (84-day) study. The study length allows observation of three potential ovulatory cycles. The upper limit of a normal ovulatory cycle length is 35 days, hence, to document ovulation using progesterone levels in the expected luteal phase, the study may extend to the end of week 14 . Ovulation will be identified by assessing the serum progesterone level on day 22 
and, if appropriate, will be repeated 1 week later. A progesterone level greater than $10 \mathrm{nmol} / \mathrm{L}$ will be used to confirm ovulation. The secondary outcome is conception (categorical "yes" or "no"). Other outcomes that will be evaluated are miscarriage rates, changes in numerical scores of depression, anxiety, stress, and quality of life based on the DASS and the FertiQoL.

\section{Statistical methods}

The participants' biochemical and hormonal profiles, physical attributes (body mass index (BMI), hirsutism), ovulatory response, pregnancy status, scores for depression, anxiety, stress and quality of life will be assessed for statistical normality. Each main effect will be compared by analysis of variance (ANOVA) or its non-parametric equivalents. The primary comparison will be between GLC and letrozole. The observed effects of myo-inositol alone are small in clinical experience and there are no reported interactions between myo-inositol and ovulation-induction medications or lifestyle change in the literature. Hence, our power calculations (see below) have been based on the two main effects acting independently. However, our factorial design will provide an opportunity to explore potential interactions (acknowledging the sample size required would change in the presence of interactions) [30, 31]. Categorical data will be compared by chi-square statistics. Differences in dietary intake and dietary composition over time will be compared by ANOVA or its non-parametric equivalent with time as the repetitive measure. Regression analysis will also be performed to determine the individual impacts of diet, activity, and mindfulness on ovulation. In addition, membership in the four PCOS sub-groups will be further explored as a potential predictor for ovulation rates in regression analysis after adjusting for BMI and age.

\section{Sample size and power calculations}

Assuming no interactions between the two main effects as discussed earlier, the target sample size will be 240 participants (120 participants in the GLC group and 120 participants on oral medication). This is based on: a power calculation using a 5\% significance level, $80 \%$ power comparing two proportions, and current knowledge of the average ovulation rates of lifestyle interventions and letrozole, our primary comparison. We explored a number of combinations of the ovulation rates from letrozole and lifestyle interventions and chose the most conservative estimate to ensure that our sample size was adequate, i.e., 91 in each group. By enrolling 120 participants in each group, we have allowed for a drop-out rate of $32 \%$. Table 1 describes the lowest and highest reported ovulation rates for letrozole and lifestyle interventions, the sample size required in each scenario comparing the two proportions, and the three
Table 1 Sample size calculation based on previous literature

\begin{tabular}{llll}
\hline $\begin{array}{l}\text { Range of reported } \\
\text { ovulation rates }\end{array}$ & $\begin{array}{l}\text { Letrozole } \\
\text { ovulation rate }\end{array}$ & $\begin{array}{l}\text { Lifestyle } \\
\text { ovulation rate }\end{array}$ & $\begin{array}{l}\text { Total sample } \\
\text { size needed }\end{array}$ \\
\hline Lowest & $62 \%^{\mathrm{a}}$ & $38 \%^{\mathrm{b}}$ & 130 \\
Highest & $86 \%^{\mathrm{c}}$ & $67 \%^{\mathrm{d}}$ & 150 \\
Median & $70 \%$ & $50 \%$ & 182 \\
\hline
\end{tabular}

aLegro et al. [29]

${ }^{\text {b} P a l o m b a ~ e t ~ a l . ~[36] ~}$

'Zeinalzadeh et al. [37]

dKarimzadeh and Javedani [38]

highest sample sizes required from our calculations, accounting for the length of lifestyle intervention and the start dose of letrozole.

When calculating the sample size necessary to assess the effect of myo-inositol on ovulation rates, we reviewed the few published studies available acknowledging that these studies all had small sample size. One randomized controlled trial reported an ovulation rate of $70 \%$ when myo-inositol was given to women with PCOS versus a $21 \%$ ovulation rate in controls [24]. Similarly, another randomized controlled trial reported an ovulation rate of $65 \%$ using myo-inositol in PCOS [32]. Using these rates in a comparison of two proportions, a total sample size of 26 is needed, and easily covered by our original calculation above.

\section{Discussion}

While previous lifestyle-related management programs can improve ovulation rates in some women with PCOS, they have generally focused on weight reduction alone. Legro et al. compared three preconception interventions for women with PCOS prior to undergoing ovulation-induction therapy. The first was lifestyle modification which consisted of caloric restriction, weight loss medication, and exercise. The second was oral contraceptive pills (OCPs) alone, and the third was lifestyle modification in combination with OCPs. The authors found that the two groups incorporating lifestyle modification achieved greater weight loss, higher rates of ovulation, and managed to avoid the onset of metabolic syndrome in comparison to the OCP group [4].

However, restrictive diets resulting in weight cycling is associated with eating disorders (as well as depression) [33]. This is concerning as women with PCOS seem to be at greater risk of developing eating disorders than the general population [34]. Furthermore, long-term weight loss medication is not a sustainable solution for ameliorating symptoms of PCOS.

The intended outcome of this research is the development of a successful comprehensive 12-week program for women with PCOS wishing to conceive. It is evident that women are seeking alternative fertility options that are natural, safe, and effective [35]. While fertility 
medications can be effective, they are costly and increase the risk of complications such as twin pregnancy. On the other hand, a lifestyle-based program can provide benefits such as weight loss, decreased symptoms of PCOS (decreased hair growth, more regular cycles), improved energy, reduced stress, and better quality of life. Patients frequently drop out of infertility treatment due to factors such as financial/emotional stress and disappointment from failure to conceive despite repeated treatments [18]. Hence, management of PCOS should be more comprehensive and address the multiple factors at play in this condition. This includes not only the endocrine and metabolic perturbations, the anovulatory infertility, but the psychological and emotional impacts of PCOS as well [19].

\section{Potential biases or limitations}

The drop-out rate is often high in lifestyle-based interventions. To mitigate a high drop-out rate we have taken steps such as providing daily email contacts and weekly check-ins between participants and health care providers throughout the duration of the study (12 weeks). A lack of compliance can often become another limitation for intervention studies. To ensure compliance of participants, especially with the lifestyle modification group, we will provide weekly in-person education sessions, as well as periodic check-ins over the phone, in order to explain and reinforce the importance and significance of the recommendations provided and how our study participants might directly benefit from such comprehensive lifestyle changes. These weekly sessions will provide an opportunity for participants to ask questions and receive support from both clinicians and other women struggling with similar issues. After each session, the participant's weight will be recorded (in a private setting) to help them remain motivated in adhering to the dietary changes and increased physical activity. Several dietary recall methods will be used to measure compliance to the low-glycemic diet, such as a 24-h recall and a food frequency questionnaire, recognizing these tools also have their own limitations.

Although the main goal of this intervention is to achieve a pregnancy, improving overall health and quality of life for women with PCOS is an important component in achieving this primary goal. Infertility per se has a significant psychological impact on women, as does the diagnosis of PCOS. Failed repetitive cycles of hormone therapy further adds to stress and reduced quality of life in PCOS, providing a rationale to develop a comprehensive support program for sustainable lifestyle changes. In addition to improving ovulatory function and the chance of conceiving, such a program has the potential to enhance long-term physical and mental health in this population.

\section{Trial status}

This trial is yet to begin recruitment. Anticipated start date is October 2018.

\section{Additional file}

Additional file 1: Standard Protocol Items: Recommendations for Interventional Trials (SPIRIT) 2013 Checklist: recommended items to address in a clinical trial protocol and related documents. (PDF $119 \mathrm{~kb}$ )

Abbreviations

DASS: Depression Anxiety Stress Scale; FertiQoL: Fertility-Related Quality of Life Tool; OCPs: Oral contraceptive pills; PCOS: Polycystic ovary syndrome

\section{Acknowledgements}

We thank Dr. Bronwyn Williams, managing director of Grace Fertility and Reproductive Medicine ("Grace Fertility Centre"), and her staff for their generous support.

Funding

There is no funding for this protocol.

Availability of data and materials

Not applicable.

\section{Authors' contributions}

$\mathrm{DAC}$ is completing her $\mathrm{PhD}$ with $\mathrm{APC}$ as her principal research supervisor. APC conceived and, with DAC, designed this trial. DAC, AKS, and APC designed the comprehensive lifestyle intervention program. APC was responsible for all sections related to clinical management, methodology, and statistical analyses. DAC wrote the manuscript and APC revised critically all earlier drafts. SMP and MAB provided advice, support and assistance. All

authors critically revised the manuscript and have approved the final version.

Ethics approval and consent to participate

This trial has been approved by the University of British Columbia Children's and Women's Research Ethics Board (H13-02964) on 22 January 2018.

Consent for publication

Not applicable.

Competing interests

The authors declare that they have no competing interests.

\section{Publisher's Note}

Springer Nature remains neutral with regard to jurisdictional claims in published maps and institutional affiliations.

Received: 4 June 2018 Accepted: 20 October 2018

Published online: 16 November 2018

References

1. Fauser BC, Tarlatzis BC, Rebar RW, Legro RS, Balen AH, Lobo R, et al. Consensus on women's health aspects of polycystic ovary syndrome (PCOS): the Amsterdam ESHRE/ASRM-sponsored 3rd PCOS consensus workshop group. Fertil Steril. 2012;97(1):38 e25.

2. Melo AS, Ferriani RA, Navarro PA. Treatment of infertility in women with polycystic ovary syndrome: approach to clinical practice. Clinics. 2015;70(11): 765-9.

3. Sozou PD, Hartshorne GM. Time to pregnancy: a computational method for using the duration of non-conception for predicting conception. PLoS One. 2012;7(10):e46544

4. Legro RS, Dodson WC, Kris-Etherton PM, Kunselman AR, Stetter CM, Williams $\mathrm{NI}$, et al. Randomized controlled trial of preconception interventions in infertile women with polycystic ovary syndrome. J Clin Endocrinol Metab. 2015;100(11):4048-58 
5. Domar AD, Seibel MM, Benson $H$. The mind/body program for infertility: a new behavioral treatment approach for women with infertility. Fertil Steril. 1990;53(2):246-9

6. Li J, Long L, Liu Y, He W, Li M. Effects of a mindfulness-based intervention on fertility quality of life and pregnancy rates among women subjected to first in vitro fertilization treatment. Behav Res Ther. 2016;77:96-104.

7. Gibson-Helm M, Teede H, Dunaif A, Dokras A. Delayed diagnosis and a lack of information associated with dissatisfaction in women with polycystic ovary syndrome. J Clin Endocrinol Metab. 2017;102(2):604-12.

8. Dokras A, Saini S, Gibson-Helm M, Schulkin J, Cooney L, Teede H. Gaps in knowledge among physicians regarding diagnostic criteria and management of polycystic ovary syndrome. Fertil Steril. 2017;107(6):1386 e1.

9. Kitzinger C, Willmott J. 'The thief of womanhood': women's experience of polycystic ovarian syndrome. Soc Sci Med. 2002;54(3):349-61.

10. Veltman-Verhulst SM, Boivin J, Eijkemans MJ, Fauser BJ. Emotional distress is a common risk in women with polycystic ovary syndrome: a systematic review and meta-analysis of 28 studies. Hum Reprod Update. 2012;18(6): 638-51.

11. Sills ES, Perloe M, Tucker MJ, Kaplan CR, Genton MG, Schattman GL. Diagnostic and treatment characteristics of polycystic ovary syndrome: descriptive measurements of patient perception and awareness from 657 confidential self-reports. BMC Womens Health. 2001;1(1):3.

12. Arentz S, Smith CA, Abbott JA, Bensoussan A. A survey of the use of complementary medicine by a self-selected community group of Australian women with polycystic ovary syndrome. BMC Complement Altern Med. 2014;14(1):1.

13. Moran LJ, Hutchison SK, Norman RJ, Teede HJ. Lifestyle changes in women with polycystic ovary syndrome. Cochrane Database Syst Rev. 2011;7(2): CD007506. https://doi.org/10.1002/14651858.CD007506.pub3.

14. Bergh CM, Moore M, Gundell C. Evidence-based management of infertility in women with polycystic ovary syndrome. J Obstet Gynecol Neonatal Nurs. 2016;45(1):111-22

15. Marsh KA, Steinbeck KS, Atkinson FS, Petocz P, Brand-Miller JC. Effect of a low glycemic index compared with a conventional healthy diet on polycystic ovary syndrome. Am J Clin Nutr. 2010;92(1):83-92.

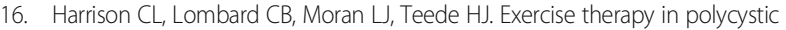
ovary syndrome: a systematic review. Hum Reprod Update. 2010;17(2):171-83.

17. Stefanaki C, Bacopoulou F, Livadas S, Kandaraki A, Karachalios A, Chrousos GP, Diamanti-Kandarakis E. Impact of a mindfulness stress management program on stress, anxiety, depression and quality of life in women with polycystic ovary syndrome: a randomized controlled trial. Stress. 2015;18(1):57-66.

18. Cousineau TM, Domar AD. Psychological impact of infertility. Best Pract Res Clin Obstet Gynaecol. 2007;21(2):293-308.

19. Berni T, Morgan C, Berni E, Rees A. Polycystic ovary syndrome is associated with adverse mental health and neurodevelopmental outcomes: a retrospective, observational study. J Clin Endocrinol Metab. 2017. https://doi. org/10.1210/jc.2017-02667.

20. Regidor P, Schindler AE. Myo-inositol as a safe and alternative approach in the treatment of infertile PCOS women: a German observational study. Int J Endocrinol. 2016;2016:1-5.

21. Unfer V, Carlomagno G, Papaleo E, Vailati S, Candiani M, Baillargeon J. Hyperinsulinemia alters myo-inositol to d-chiroinositol ratio in the follicular fluid of patients with PCOS. Reprod Sci. 2014;21(7):854-8.

22. Milewska EM, Czyzyk A, Meczekalski B, Genazzani AD. Inositol and human reproduction. from cellular metabolism to clinical use. Gynecol Endocrinol. 2016;32(9):690-5.

23. Chiu TT, Rogers MS, Law EL, Briton-Jones CM, Cheung LP, Haines CJ. Follicular fluid and serum concentrations of myo-inositol in patients undergoing IVF: Relationship with oocyte quality. Hum Reprod. 2002;17(6): 1591-6.

24. Costantino D, Minozzi G, Minozzi E, Guaraldi C. Metabolic and hormonal effects of myo-inositol in women with polycystic ovary syndrome: a doubleblind trial. Eur Rev Med Pharmacol Sci. 2009;13(2):105-10.

25. Pizzo A, Laganã AS, Barbaro L. Comparison between effects of myo-inositol and D-chiro-inositol on ovarian function and metabolic factors in women with PCOS. Gynecol Endocrinol. 2014;30(3):205

26. Ciotta L, Stracquadanio M, Pagano I, Carbonaro A, Palumbo M, Gulino F. Effects of myo-inositol supplementation on oocyte's quality in PCOS patients: a double blind trial. Eur Rev Med Pharmacol Sci. 2011;15(5):509-14.

27. Boivin J, Takefman J, Braverman A. Development and preliminary validation of the fertility quality of life (FertiQoL) tool. Hum Reprod. 2011;26(8):2084-91.
28. Lovibond SH, Lovibond PF. Manual for the Depression Anxiety Stress Scales. 2nd ed. Sydney: Psychology Foundation; 1995.

29. Legro RS, Brzyski RG, Diamond MP, Coutifaris C, Schlaff W, Casson P, et al. Letrozole versus clomiphene for infertility in the polycystic ovary syndrome. N Engl J Med. 2014;371(2):119-29.

30. McAlister FA, Straus SE, Sackett DL, Altman DG. Analysis and reporting of factorial trials: a systematic review. JAMA. 2003;289(19):2545-53.

31. Jaki T, Vasileiou D. Factorial versus multi-arm multi-stage designs for clinical trials with multiple treatments. Stat Med. 2017;36:563-80.

32. Raffone E, Rizzo P, Benedetto V. Insulin sensitizer agents alone and in cotreatment with r-FSH for ovulation induction in PCOS women. Gynecol Endocrinol. 2010;26(4):275-80.

33. Yanovski SZ. Dieting and the development of eating disorders in overweight and obese adults. Arch Intern Med. 2000;160(17):2581-9.

34. Himelein MJ, Thatcher SS. Polycystic ovary syndrome and mental health: a review. Obstet Gynecol Surv. 2006;61(11):723-32.

35. Raja-Khan N, Stener-Victorin E, Wu X, Legro RS. The physiological basis of complementary and alternative medicines for polycystic ovary syndrome. Am J Physiol Endocrinol Metab. 2011;301(1):E10.

36. Palomba S, Falbo A, Giallauria F, Russo T, Rocca M, Tolino A, et al. Six weeks of structured exercise training and hypocaloric diet increases the probability of ovulation after clomiphene citrate in overweight and obese patients with polycystic ovary syndrome: a randomized controlled trial. Hum Reprod. 2010;25:2783-91.

37. Zeinalzadeh M, Basirat Z, Esmailpour M. Efficacy of letrozole in ovulation induction compared to that of clomiphene citrate in patients with polycystic ovarian syndrome. J Reprod Med. 2010;55(1-2):36-40.

38. Karimzadeh MA, Javedani M. An assessment of lifestyle modification versus medical treatment with clomiphene citrate, metformin, and clomiphene citrate-metformin in patients with polycystic ovary syndrome. Fertil Steril. 2010;94(1):216-20.

\section{Ready to submit your research? Choose BMC and benefit from:}

- fast, convenient online submission

- thorough peer review by experienced researchers in your field

- rapid publication on acceptance

- support for research data, including large and complex data types

- gold Open Access which fosters wider collaboration and increased citations

- maximum visibility for your research: over $100 \mathrm{M}$ website views per year

At $\mathrm{BMC}$, research is always in progress.

Learn more biomedcentral.com/submissions 